\title{
Preprocessing by a Bayesian Single-Trial Event-Related Potential Estimation Technique Allows Feasibility of an Assistive Single-Channel P300-Based Brain-Computer Interface
}

\author{
Anahita Goljahani, ${ }_{1}^{1}$ Costanza D'Avanzo, ${ }^{1}$ Stefano Silvoni, ${ }^{2}$ Paolo Tonin, ${ }^{2}$ \\ Francesco Piccione, ${ }^{2}$ and Giovanni Sparacino ${ }^{1}$ \\ ${ }^{1}$ Department of Information Engineering, University of Padova, Via Gradenigo 6/B, 35131 Padova, Italy \\ ${ }^{2}$ I.R.C.C.S. San Camillo Hospital Foundation, Via Alberoni 70, 30126 Venice, Italy
}

Correspondence should be addressed to Giovanni Sparacino; gianni@dei.unipd.it

Received 17 April 2014; Revised 18 June 2014; Accepted 18 June 2014; Published 7 July 2014

Academic Editor: Fabio Babiloni

Copyright ( $) 2014$ Anahita Goljahani et al. This is an open access article distributed under the Creative Commons Attribution License, which permits unrestricted use, distribution, and reproduction in any medium, provided the original work is properly cited.

\begin{abstract}
A major clinical goal of brain-computer interfaces (BCIs) is to allow severely paralyzed patients to communicate their needs and thoughts during their everyday lives. Among others, P300-based BCIs, which resort to EEG measurements, have been successfully operated by people with severe neuromuscular disabilities. Besides reducing the number of stimuli repetitions needed to detect the P300, a current challenge in P300-based BCI research is the simplification of system's setup and maintenance by lowering the number $N$ of recording channels. By using offline data collected in 30 subjects (21 amyotrophic lateral sclerosis patients and 9 controls) through a clinical BCI with $N=5$ channels, in the present paper we show that a preprocessing approach based on a Bayesian single-trial ERP estimation technique allows reducing $N$ to 1 without affecting the system's accuracy. The potentially great benefit for the practical usability of BCI devices (including patient acceptance) that would be given by the reduction of the number $N$ of channels encourages further development of the present study, for example, in an online setting.
\end{abstract}

\section{Introduction}

Brain-computer interfaces (BCIs) are cutting-edge systems aimed at identifying subjects' intention from measurements of brain activity [1-3]. A major clinical challenge in BCI research has been to develop systems capable of restoring communication in those people who, because of brainstem strokes, cerebral palsies, brain/spinal cord injuries, or progressive neurodegenerative diseases (such as amyotrophic lateral sclerosis, ALS), have lost the control of nearly all voluntary muscles but still retain cognition and sensation $[4,5]$.

Noninvasiveness, high temporal resolution, and low encumbrance are among the reasons why EEG-based BCIs are considered particularly appealing for the development of systems intended to be finally used at patients' home $[3,6]$. The literature is huge and here is sufficient to mention that in severely paralyzed or disabled patients EEG-based BCIs have been successfully operated by exploiting slow cortical potentials [7, 8], sensorimotor $\mu$ and $\beta$ rhythms [9-12], the P300 component of event-related potentials (ERPs) [13$20]$, and the electrical activity associated with semantically conditioned mental responses [21].

While some of the above-cited control signals may require long trainings for being reliably produced accordingly with users' intention, for example, several weeks in $[7,10]$, the P300 requires only focused attention and a suitable stimulation scheme for being generated, approximately $300 \mathrm{~ms}$ after a rare or task-relevant (target) stimulus [22]. Since the ERP is embedded in background EEG activity, the target stimulus has to be repeated several times in order to detect the P300, often from the average of $M$ EEG epochs aligned with the stimulus onset $[16,19]$. However, reliable generation and detection of the P300 with as few as possible stimulus repetitions (trials) is needed for obvious practical reasons. To this aim, BCI investigators have studied ad hoc stimulation 
schemes [20, 23-25], optimal channel sets [14, 26], channel selection methods [27, 28], classification algorithms [29-32], and spatial filtering approaches [18, 33-35]. These efforts led to the development of P300-based BCIs reliably operable by a very small number of repetitions $(\sim 4$ in [20] with $N=$ 8 channels), and, in few cases, even to single-trial systems, obtained, for example, by spatially filtering data from $N=5$ recording channels $[13,17]$.

Yet, speed and accuracy are not the only concerns in BCI research. Particularly in clinical applications, user's physical condition may render simplicity of use and setup even more important than a theoretical $100 \%$ accuracy [36], as witnessed, for instance, by the fact that a patient involved in the study reported in [16] decided to withdraw from the investigation because attaching and maintaining too many electrodes was considered unacceptable. This is, indeed, a general concern shared by BCI users and their caregivers [37] and, although six to eight channels were believed to be optimal for classification accuracy in P300-based BCIs $[26,30]$, reduction of channels is advocated [16].

In this paper, we consider the challenge of reducing to one the number $N$ of channels in a single-trial P300-based BCI. Specifically, as a preliminary proof of concept of the feasibility of an assistive single-channel (SC) BCI, we assess the hypothesis that the performance of the multichannel (MC) BCI system documented in [13], with $N=5$, is preserved when only one channel is employed but a Bayesian ERP estimation technique $[38,39]$ is used to preprocess the signal. For such a scope, an offline comparison is made by using the data collected in 21 ALS patients and 9 healthy controls. Results will show that, in terms of classification accuracy, the performance of the SC BCI is not significantly different from that of the MC BCI, published in [13].

The paper is organized as follows. In Section 2, after a brief review of experimental protocol and reference MC BCI used in [13], the algorithms of the SC BCI prototype are described. Accuracy of the SC and MC systems is assessed and compared in Section 3. Comments on results, practical challenges, limits of the study, and margins for further investigations are reported in Section 4. Some conclusions end the paper in Section 5.

\section{Materials and Methods}

2.1. Experimental Protocol and Database. Data utilized in the present study for offline analyses are those recorded during the online BCI sessions described in [13] from 21 ALS patients (aged $55.6 \pm 14.3$ years), in early and middle stages of the disease $(32.2 \pm 6.7$ ALSFRS-R score [40], $47 \pm 32$ months from the disease onset), and 9 healthy controls (aged $54 \pm 18.8$ years). We refer the reader to [13] for details. Briefly, users were faced with a monitor with four images at its borders, representing four basic needs, for example, being hungry, and a circle in the center, as shown in Figure 1. The stimulation consisted of consecutive blocks of randomized flashings of four arrows (upward, rightward, downward, and leftward), each pointing to the direction of one image. By focusing on the flashing of the arrow pointing to the desired image (target stimulus) and ignoring the others (nontarget stimuli),

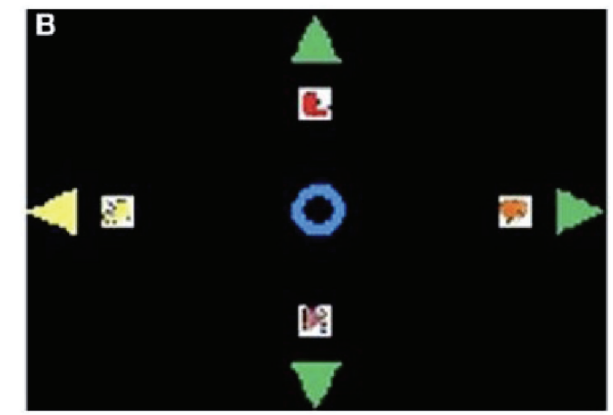

FIGURE 1: Graphical interface of the reference P300-based BCI [13]. The four icons represent four different basic needs. The flashing arrow on the left side is an example of stimulation.

a P300 component was elicited. EEG was recorded from four channels, that is, $\mathrm{Fz}, \mathrm{Cz}, \mathrm{Pz}$, and $\mathrm{Oz}$, and the electrooculogram (EOG) was recorded from two electrodes placed laterally and below the left eye. All electrodes were referenced to the left earlobe. Signals were amplified by a SynAmps (NeuroSoft, Inc.) amplifier, band-pass filtered between 0.15 and $30 \mathrm{~Hz}$, digitized with a 16-bit resolution, and sampled at $200 \mathrm{~Hz}$. After each flashing, a detection of a P300 activity from the measured EEG determined the movement of the circle by one step towards the direction of the flashed arrow and four consecutive steps in the desired direction were needed to reach the image. The time interval between two consecutive flashings, that is, the inter stimulus interval (ISI), was $2.5 \mathrm{~s}$. Each BCI session started with the circle at the center of the screen and ended when the user reached the desired image or a time-out occurred (defined below for testing sessions only).

Sessions were distributed over five days. In the first day, eight sessions were carried out to collect data for initial calibration tasks. In particular, target and nontarget EEG data were recorded with an automatic feedback; that is, after each target flashing, the system automatically moved the circle towards the target image and, after each nontarget flashing, the system kept the circle still. In each automatic-feedback session, the number of flashings varied from a minimum of 13 to a maximum of 16, depending on when the target arrow was flashed in the fourth randomized block. In each of the subsequent testing days, denoted as T1, T2, T3, and T4, four sessions with feedback based on the identification of the P300 by means of the classification algorithm were carried out. Each testing session ended when the user reached the desired image or after a maximum of 92 flashings, corresponding to a time-out of 3 minutes and 50 seconds.

A classification error in correspondence with a nontarget stimulus determined the movement of the circle towards the wrong image, whereas a classification error in correspondence with a target stimulus determined the lack of a movement towards the desired image.

2.2. The Reference Multichannel (MC) BCI. EEG raw epochs, starting $500 \mathrm{~ms}$ before and ending $1000 \mathrm{~ms}$ after each flashing, were extracted and baseline was corrected to the mean of prestimuli data, resulting in $n_{\text {pre }}=100$ prestimulus and $n_{\text {post }}=200$ poststimulus samples. Functional blocks of 


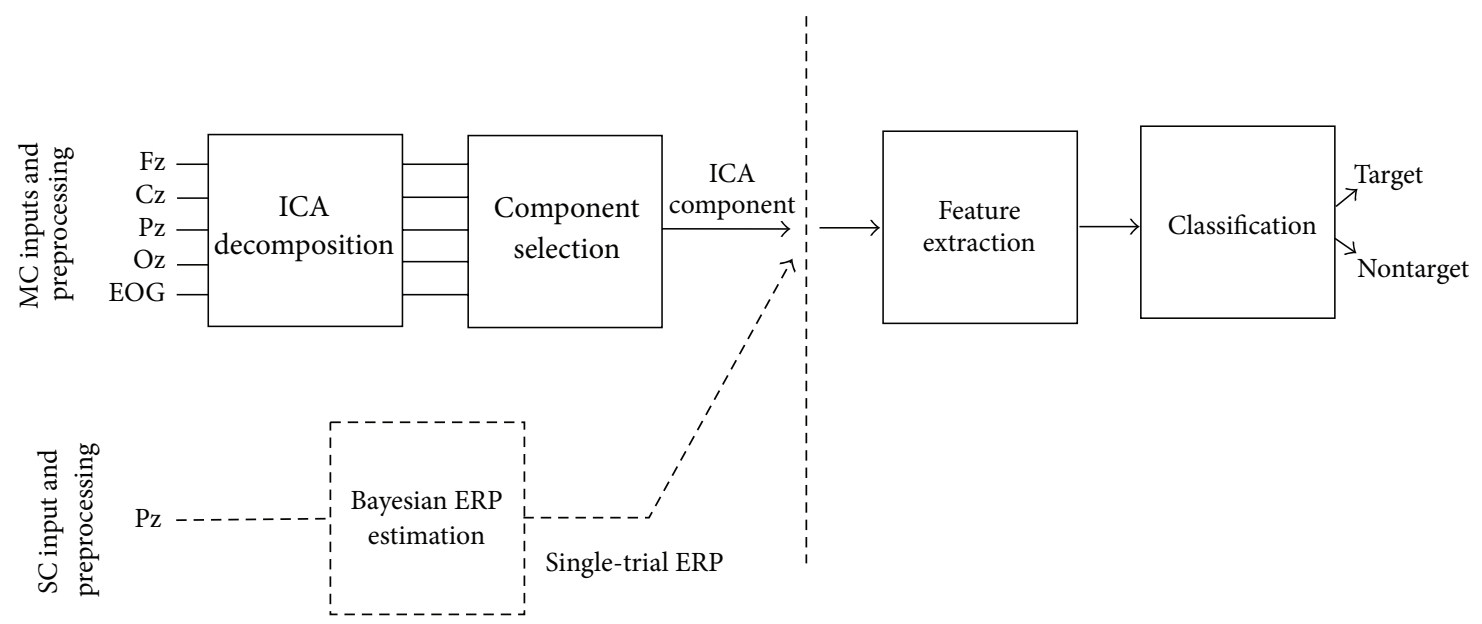

FIGURE 2: Functional blocks of the reference MC BCI and of the simulated SC prototype. MC (solid lines) and SC (dashed lines) preprocessing blocks are highlighted on the left side.

the reference multichannel (MC) BCI [13] are graphically illustrated in Figure 2. Briefly, in correspondence with each flashing, raw epochs from $N=5$ channels, that is, $\mathrm{Fz}, \mathrm{Cz}, \mathrm{Pz}$, $\mathrm{Oz}$, and EOG, fed a single-trial ICA decomposition block that produced five independent components. One of the components was, then, selected and used to extract the information (features) supplied to the classifier to take the decision about whether the stimulus that produced the signal was target or nontarget. Seventy-eight features were computed for each epoch and comprised, for example, latencies and values of minimum and maximum peaks, power of the signal in $200 \mathrm{~ms}$ windows, and wavelet coefficients [13, 17]. Feature vectors were classified by means of a support vector machine (SVM) classifier with a radial basis function kernel [41].

As explained in more detail in [13], calibration tasks were performed at the beginning of each testing day using data collected during all preceding BCI sessions. Specifically, based on calibration data, for each subject, the ICA demixing matrix and the index of the component to be selected were determined offline by means of the algorithms described in $[42,43]$, respectively. Moreover, parameters needed for running the SVM classifier were derived by a cross-validation procedure on sets obtained by repeatedly splitting calibration data in $80 \% / 20 \%$ fractions.

2.3. The Single-Channel (SC) BCI Prototype. The singlechannel (SC) BCI prototype that we simulate and assess offline in the present paper is obtained by replacing the preprocessing steps of the MC BCI (blocks with solid lines in the left portion of Figure 2), which exploits 5 inputs, with a single-trial ERP estimation algorithm operating only on the Pz channel (block with dashed lines in Figure 2). Singletrial ERPs are estimated through the Bayesian approach extensively described in [38], which also admits further sophistications, recently documented in $[39,44]$, though not usable in real time and thus unsuited to the BCI setting.

Briefly, the method performs an ad hoc smoothing of each EEG raw epoch by exploiting, in a Bayesian setting, prior knowledge on the smoothness of the unknown ERP, described as $m$ discrete integrations of a white noise with variance $\lambda^{2}$, and on the autocorrelation of the background EEG (noise), obtained from an AR model of order $p$ with coefficients and variance parameter $\sigma^{2}$ identified from the prestimulus data of each epoch. In our application, $m=2$, while $p$ is determined, according to the final predictor error (FPE) criterion, epoch by epoch. $\lambda^{2}$ being unknown, the ratio $\gamma=\sigma^{2} / \lambda^{2}$, which determines the single-trial smoother, is determined, epoch by epoch, by the popular discrepancy regularization criterion [45]. An example of application of the single-trial ERP estimation to our BCI data is described in Section 3.1.

After $\mathrm{Pz}$ data preprocessing, the same features defined and employed in the reference MC BCI are extracted (from the estimated single-trial ERPs) and, finally, classified by an SVM classifier with the same type of kernel. In order to allow a fair comparison between SC and $\mathrm{MC}$, available datasets were processed offline by the $\mathrm{SC} \mathrm{BCI}$ in the same way as in the online MC BCI. Specifically, for each subject, before processing single-trial data from each testing day, calibration tasks, for example, classifier training, were performed based on $\mathrm{Pz}$ datasets from all preceding sessions. This was done to obtain, for each subject and testing day, accuracy comparable to that obtained by the MC BCI and assess the role of the proposed preprocessing approach on the system's performance.

Remark. The determination, epoch by epoch, of $\gamma$ can be computationally expensive (see [46]). While this is not an issue for offline calibration tasks, it can affect online operability. For this reason, in the present paper, for each testing session, instead of optimizing $\gamma$ for each epoch, we utilize a fixed $\gamma^{*}$ predetermined from calibration data as the median of the set of $\gamma$ values "optimally" tuned, for all target and nontarget calibration epochs, by the discrepancy criterion.

\section{Results}

3.1. Output of the New Pre-Processing Step. Before investigating the performance of the BCI system, it is useful to 


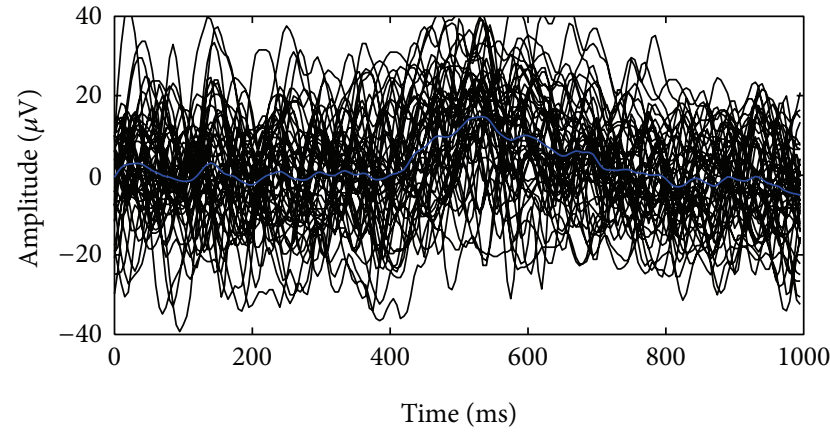

(a) Raw target epochs

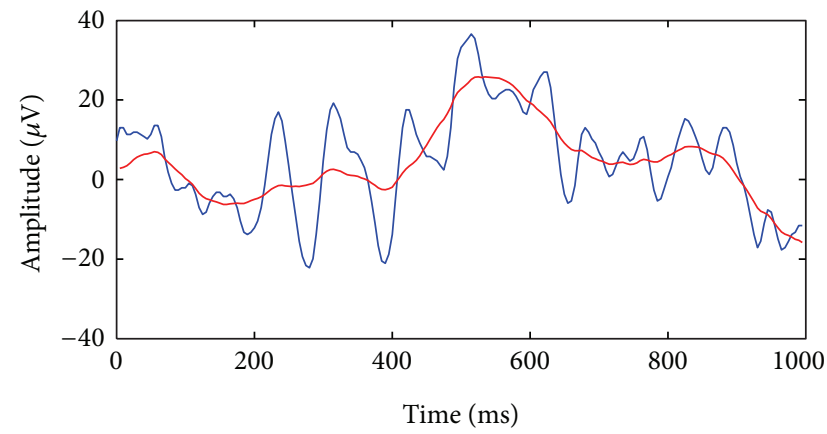

- Raw

- Preprocessed

(c) Target number 41

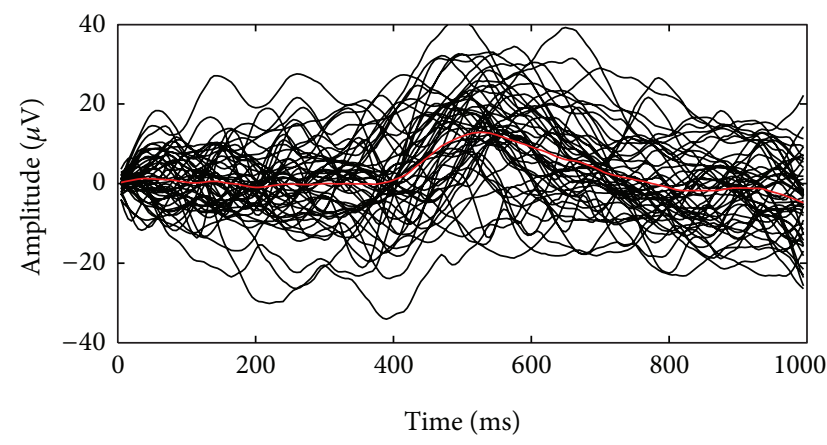

(e) Preprocessed target epochs

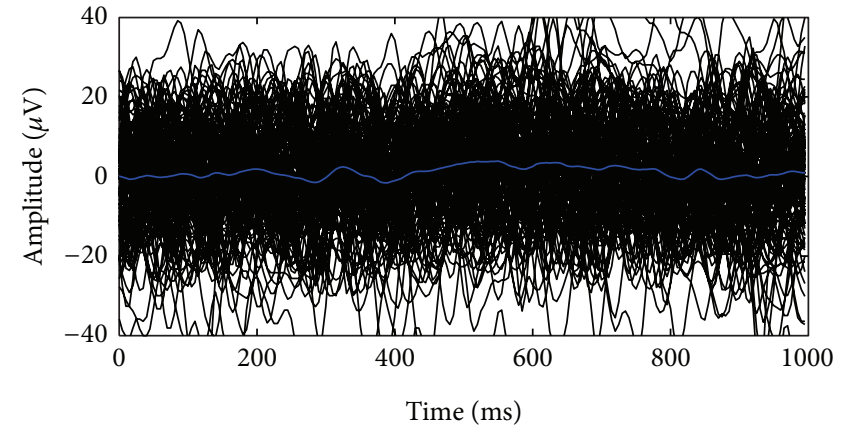

(b) Raw nontarget epochs

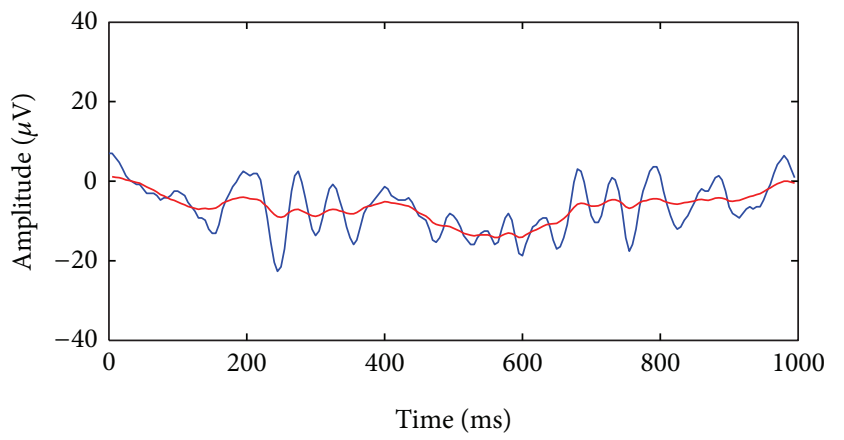

Raw
— Preprocessed

(d) Nontarget number 4

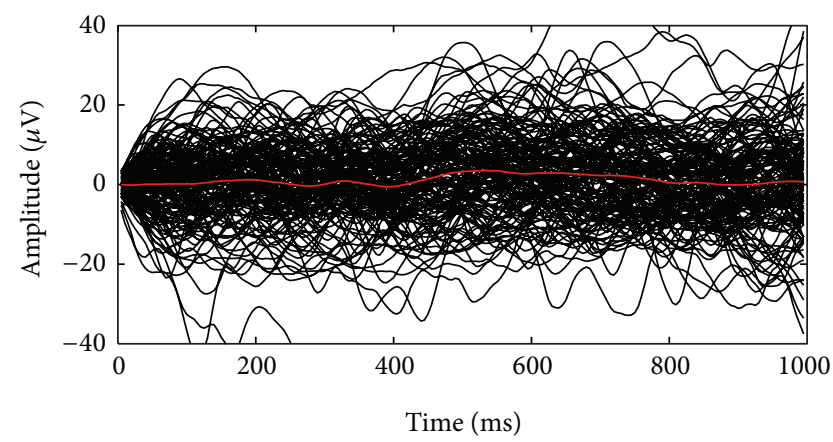

(f) Preprocessed nontarget epochs

FIGURE 3: Preprocessing results for a representative ALS patient. Raw target and nontarget epochs collected from P8 in T4 are shown in (a) and (b), respectively, together with their average, shown as solid blue lines. In (c) and (d) two representative raw target and nontarget epochs (blue curves) are superimposed to their denoised versions obtained by the Bayesian preprocessing (red curves). In (e) and (f) signals obtained by preprocessing target and nontarget epochs in (a) and (b), respectively, together with their averages (red curves), are shown.

show an example of application of the single-trial estimation technique to our data. Figure 3 reports, in panels (a) and (b), respectively, single-trial target and nontarget raw epochs recorded from $\mathrm{Pz}$ in patient $\mathrm{P} 8$ during the testing day $\mathrm{T} 4$. The blue curves drawn in the same panels are the averages of target and nontarget raw epochs, respectively. Panels (c) and (d) display results of the new preprocessing step (red curves) for one representative target and one representative nontarget raw epoch (blue curves). Finally, the black curves in panels (e) and (f) are the preprocessed versions of all curves in panels (a) and (b), respectively, and the red curves are their averages.
As visible from the blue curves in panels (a) and (b), target raw epochs are characterized by an average positive deflection that is not present in nontarget epochs. The deflection, which takes place at around $500 \mathrm{~ms}$, is the P300 component of the ERP. The red curve in panel (c) shows that, at the single-trial level, the considered preprocessing smoothes away spurious oscillations and produces a signal in which the P300-related activity is more evident. As far as nontarget epochs are concerned, panel (d) confirms that, as expected, the proposed preprocessing yields a signal that is quite flat, reflecting the absence of a P300-related activity. Finally, panels (e) and (f) 
TABLE 1: Percentage accuracy achieved by ALS patients in testing days T1, T2, T3, and T4 by means of the MC BCI (MC acc.) and the SC BCI prototype (SC acc.).

\begin{tabular}{|c|c|c|c|c|c|c|c|c|}
\hline & \multicolumn{2}{|c|}{$\mathrm{T} 1$} & \multicolumn{2}{|c|}{$\mathrm{T} 2$} & \multicolumn{2}{|c|}{ T3 } & \multicolumn{2}{|c|}{$\mathrm{T} 4$} \\
\hline & MC acc. (\%) & SC acc. (\%) & MC acc. (\%) & SC acc. (\%) & MC acc. (\%) & SC acc. (\%) & MC acc. (\%) & SC acc. (\%) \\
\hline $\mathrm{P} 1$ & 73,5 & 72,6 & 73 & 78,4 & 78,3 & 81,7 & 77,9 & 81 \\
\hline P2 & 60,3 & 71,3 & 81,3 & 76,1 & 74,2 & 71,4 & 70,8 & 75,2 \\
\hline P3 & 63,4 & 69,2 & 90,9 & 69 & 80,6 & 70,5 & 87,2 & 74,1 \\
\hline P4 & 65 & 74,9 & 69,5 & 73,4 & 61,6 & 74,9 & 75,3 & 73,5 \\
\hline P5 & 80,2 & 78,8 & 80,6 & 70,6 & 89 & 83,7 & 82,1 & 82 \\
\hline P6 & 80,9 & 78,6 & 81,3 & 86,2 & 86,4 & 89,3 & 88,7 & 85,2 \\
\hline P7 & 72,9 & 80,7 & 76,2 & 75,4 & 74,7 & 79,2 & 72,7 & 80,1 \\
\hline P8 & 76,6 & 86,1 & 78,6 & 83,7 & 80,6 & 76,7 & 76,3 & 83,7 \\
\hline P9 & 80,4 & 78,6 & 78,4 & 90,2 & 79,1 & 88,7 & 84,4 & 83,9 \\
\hline P10 & 82,2 & 76,2 & 77,9 & 79 & 80,1 & 85,6 & 79,4 & 87,5 \\
\hline P11 & 82 & 72,3 & 78,8 & 78,5 & 74,7 & 76,5 & 85,2 & 69,7 \\
\hline $\mathrm{P} 12$ & 79,1 & 76,5 & 85,7 & 75,5 & 70,7 & 81,5 & 79,3 & 77 \\
\hline $\mathrm{P} 13$ & 77,6 & 77,6 & 86,4 & 86,3 & 88,4 & 83,1 & 91 & 96,7 \\
\hline P14 & 75,9 & 72,9 & 79 & 74 & 85,4 & 83,2 & 77,2 & 81,5 \\
\hline P15 & 67 & 87,5 & 79 & 95,1 & 79,2 & 90,7 & 80,3 & 89,7 \\
\hline P16 & 61,5 & 63,9 & 56,4 & 74,8 & 79,4 & 78,4 & 76,8 & 83,7 \\
\hline P17 & 70,6 & 70,4 & 75,3 & 76,6 & 80 & 74,2 & 71,4 & 70,9 \\
\hline P18 & 66,4 & 70,3 & 74,6 & 74,8 & 80,9 & 79,5 & 78 & 80 \\
\hline P19 & 78,1 & 82,5 & 88,8 & 85,3 & 84,3 & 85,9 & 69,6 & 84,9 \\
\hline P20 & 72,9 & 72,4 & 77,5 & 70 & 86,4 & 76,8 & 78,9 & 74,1 \\
\hline P21 & 72,7 & 72,3 & 69,5 & 76,9 & 75,5 & 82,9 & 77,4 & 79,3 \\
\hline
\end{tabular}

show how the extracted activity varies from epoch to epoch, with average activities (red curves) similar to the ones of raw epochs.

Similar comments could be drawn from the results obtained in the other patients and in healthy subjects, irrespectively of ERP interepoch variations and general group differences, visible, for example, from the grand averages depicted in Figure 4, which show a lower and slightly delayed P300 for patients with respect to controls. Indeed, according to the comprehensive simulation studies reported in $[38,39]$, the accuracy of ERP estimates depends on the signal-to-noise ratio (SNR) and not on the specific shape of the ERP.

3.2. Assessment of Classification Accuracy: SC versus MC. Classification accuracy obtained by feeding the SC prototype with the input data described in Section 2.1, together with the reference accuracy published in [13], is reported in Tables 1 and 2 for ALS patients and controls, respectively. Results are grouped by testing day and, for brevity, accuracy by the MC BCI of [13] and by the SC BCI prototype is denoted as "MC accuracy" and "SC accuracy," whereas labels P1,.., P21 and $\mathrm{H} 1, \ldots, \mathrm{H} 9$ are used for patients and controls, respectively.

Numerical results in Tables 1 and 2 are graphically represented in Figure 5, where a series of box-plots are reported in two panels related to ALS patients (panel (a)) and healthy controls (panel (b)), respectively. Each box-plot is based on values from one column of the tables, that is, on the accuracy achieved in a specific testing day, $\mathrm{T}_{i}, i=1, \ldots, 4$,

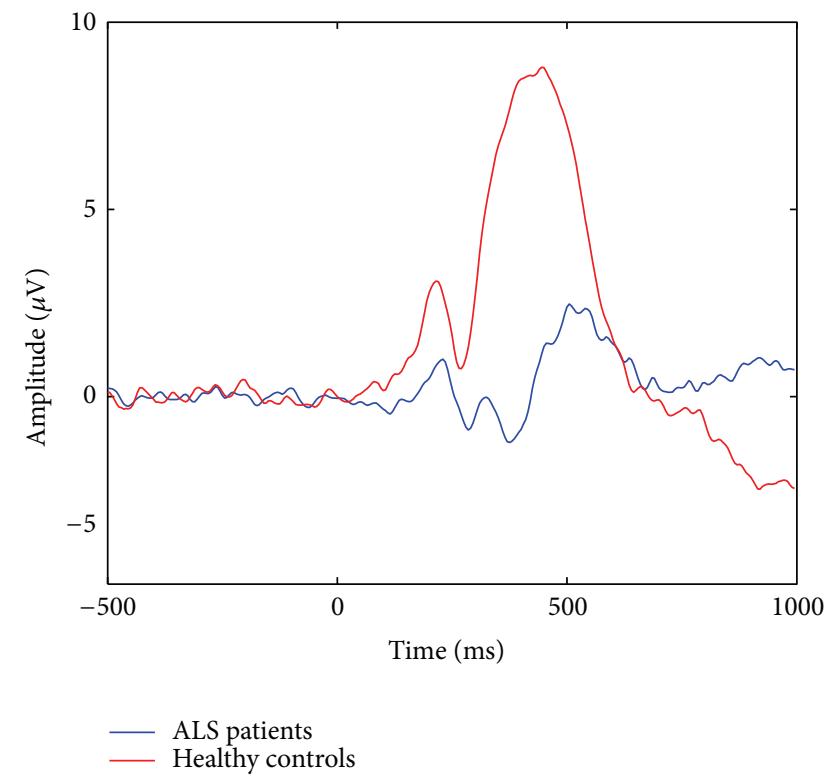

Figure 4: Average of target epochs collected from $\mathrm{Pz}$ during all testing days for ALS patients (red curve) and healthy controls (blue curve).

and by a specific system, MC or SC. The lower and upper edges of the rectangles are drawn in correspondence with the 25 th and 75th percentile of accuracy, respectively, and the red lines represent the median accuracy. 
TABLE 2: Percentage accuracy achieved by healthy controls in testing days T1, T2, T3, and T4 by means of the MC BCI (MC acc.) and the SC $\mathrm{BCI}$ prototype (SC acc.).

\begin{tabular}{|c|c|c|c|c|c|c|c|c|}
\hline & \multicolumn{2}{|c|}{$\mathrm{T} 1$} & \multicolumn{2}{|c|}{$\mathrm{T} 2$} & \multicolumn{2}{|c|}{$\mathrm{T} 3$} & \multicolumn{2}{|c|}{$\mathrm{T} 4$} \\
\hline & MC acc. (\%) & SC acc. (\%) & MC acc. (\%) & SC acc. (\%) & MC acc. (\%) & SC acc. (\%) & MC acc. (\%) & SC acc. (\%) \\
\hline $\mathrm{H} 1$ & 73,6 & 81,9 & 77,3 & 84,1 & 73,9 & 84,5 & 79,6 & 84,5 \\
\hline $\mathrm{H} 2$ & 73,3 & 81,2 & 77,5 & 86,5 & 86,6 & 91,8 & 78,6 & 91,9 \\
\hline $\mathrm{H} 3$ & 71,2 & 74,6 & 76,9 & 76,8 & 71,8 & 76 & 76,1 & 75 \\
\hline $\mathrm{H} 4$ & 85,9 & 87 & 80,8 & 89 & 86,3 & 86,7 & 89,4 & 94,1 \\
\hline H5 & 81,2 & 77,6 & 91,3 & 79,12 & 81 & 77,6 & 87,4 & 84,6 \\
\hline H6 & 76,4 & 79,6 & 83,1 & 80,6 & 81,9 & 79,8 & 78,2 & 73,1 \\
\hline $\mathrm{H} 7$ & 95,1 & 87,6 & 86,9 & 81,7 & 89 & 88,1 & 93,1 & 83,5 \\
\hline $\mathrm{H} 8$ & 70,1 & 75,8 & 83,6 & 68,9 & 76,1 & 77,9 & 83,5 & 76,7 \\
\hline $\mathrm{H} 9$ & 77,3 & 86,1 & 75,7 & 72,4 & 70,3 & 82 & 80,7 & 92 \\
\hline
\end{tabular}

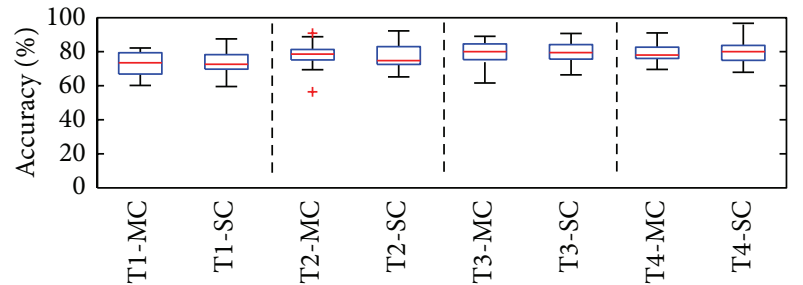

(a) ALS patients

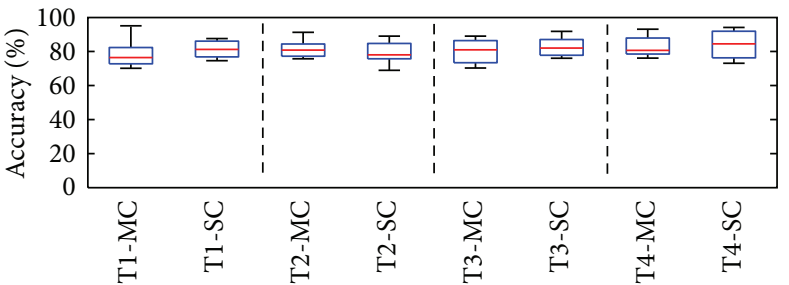

(b) Controls

FIGURE 5: Distributions of accuracy achieved by MC and SC systems in T1, .., T4 for ALS patients (a) and controls (b). Labels Tj-MC and $\mathrm{T} j$-SC for, $j=1, \ldots, 4$, denote accuracy achieved in testing day T $j$ by MC and SC systems, respectively.

As shown by the plots, in all testing days the median accuracy yielded by the two systems is very close both for patients and for controls. Actually, based on values in Tables 1 and 2 , it can be assessed that differences between medians are below $5 \%$ and, whereas the average performance in $\mathrm{T} 1$ and $\mathrm{T} 2$ is slightly higher when the MC BCI is employed, the inverse happens in T3 and T4, where the SC prototype yields slightly higher medians for both groups, for example, $81 \%$ versus $78 \%$ and $84,5 \%$ versus $80,7 \%$ for patients and controls, respectively, in T4. The plots in Figure 5 also show that for all testing days the distributions of accuracy around the medians mainly overlap for the two systems, without a clear predominance of one system over the other. For instance, in T4 the accuracy achieved by ALS patients is concentrated between $76,1 \%$ and $82,7 \%$ for the MC BCI and between $74,9 \%$ and $83,7 \%$ for the SC prototype. Similarly, for healthy controls, the ranges are $78,5-87,9 \%$ and $76,3-91,9 \%$, respectively. In order to validate these graphical evidences, MC accuracy and SC accuracy were compared by means of a statistical test. Specifically, both for patients and for controls, a Wilcoxon paired test was performed on accuracy from each testing day. None of the tests revealed statistically significant differences, confirming that, thanks to the proposed preprocessing, users' intention can potentially be decodable from only one channel with the same accuracy achieved with the reference $N=5$ channels BCI. As a final remark, in terms of best cases, it is interesting to note that maximum accuracy achieved by ALS patients in each testing day by means of the SC prototype, that is, $87.5 \%$, $92.2 \%, 90.7 \%$, and $96.7 \%$, in $\mathrm{T} 1, \mathrm{~T} 2, \mathrm{~T} 3$, and $\mathrm{T} 4$, respectively, besides being impressively high from the first day, slightly outperforms that achieved by the MC BCI, that is, $82.2 \%$, $90.9 \%, 89 \%$, and $91 \%$, with the best improvement of $5.7 \%$ obtained in T4. The same happens for healthy controls in T3 and T4, where the SC prototype yields maximum accuracy of $91,8 \%$ and $94,1 \%$, respectively, versus the $89 \%$ and $93,1 \%$ of the MC BCI. It is worthy to note that all users only participated in the five-day experimental protocol described in Section 2.1.

\section{Discussion}

Offline analysis on data collected in 21 ALS patients and 9 controls indicates that the proposed Bayesian preprocessing technique yields signals with enhanced SNR well evidencing the presence or absence of P300-related activity. Accuracy of the SC prototype was found to be not statistically different from that of the MC BCI, confirming that no performance degradation was caused by the reduction of channels from $N=5$ to $N=1$.

Remarkably, results about SC accuracy, reported in Section 3.2, were obtained without any strategy to individually select the single channel. Interesting insights in this regard may be obtained from an accurate inspection of the outcomes in some specific cases (details not reported for the sake of brevity of the paper). For instance, in those cases in 
which SC performed worst, we observed that Pz did not show an evident P300 activity and that an improvement in accuracy could be achieved by using data from $\mathrm{Cz}$, for example, from $67.9 \%$ to $80.7 \%$ for patient P11 in T4. Similar examples were found that highlighted the benefit of an optimized electrode positioning in SC systems.

As far as communication speed is concerned, the $2.5 \mathrm{~s}$ ISI of the considered four choices paradigm yields an upper bound of $12 \mathrm{bit} / \mathrm{min}$ for the information transfer bit rate (TBR), computed as in [17]. Interestingly, this value is almost achieved by P15 in T2, where the SC prototype allows a communication of $11.2 \mathrm{bit} / \mathrm{min}$.

Limits of the present study include the fact that results were obtained in an offline setting, where adaptation mechanisms that take place when the user receives the feedback to his/her mental activity cannot be reproduced. Therefore, an online implementation of the SC prototype will be needed to validate and confirm the results obtained in the simulated environment of this paper. Another potentially critical point concerns the ISI, which should be reduced in order to increase communication speed. Obviously, short ISIs, for example, the $400 \mathrm{~ms}$ proposed in [14] for a six-icon paradigm, determine a possible spread of the target P300 activity in epochs corresponding to nontarget stimuli. Proper investigations would be needed in this case to assess the capability of the classifier to predict users' intention from the activity estimated by the Bayesian technique. In any case, a short ISI would require improving numerical implementation of the Bayesian preprocessing algorithm. In particular, in place of the matrix-vector approach [38] used also in the present paper, methods implementing Wiener filtering by $Z$ transforms and spectral factorization techniques [47] should be chosen.

Finally, it is worth mentioning that the number of ALS datasets available for the present work, that is, 21, is quite high with respect to commonly published BCI investigations. In perspective, this adds to the potential clinical interest of the study, because, sometimes, results obtained in healthy subjects do not reflect those of patients [48].

\section{Conclusions}

The study presented in this paper shows that a preprocessing by a sophisticated single-trial ERP estimation technique can potentially allow driving a P300-based BCI by a single channel. This can be of high impact in BCI clinical applications, where simplicity of use and setup can be even more important than $100 \%$ of accuracy and reduction of the number $N$ of channels has been solicited [16]. Moreover, a reduced number of channels would also have an impact on power consumption in wireless EEG caps.

As future developments of the present study, besides validating results in an online setting and with diverse stimulation schemes with variable ISIs, further research will be aimed at better understanding of to what extent singletrial ERP estimation techniques may effectively contribute to improve P300-based BCI systems, for example, by optimizing the position of the single channel. A further boost to the practical usability of P300-based BCIs may be given by the use of a subcutaneous (minimally invasive) electrode such as that proposed in [49] for hypoglycemia detection.

\section{Conflict of Interests}

The authors declare that there is no conflict of interests regarding the publication of this paper.

\section{Acknowledgment}

The authors are grateful to Ms. C. Genna for the support given in preliminary studies about the topic of the present paper.

\section{References}

[1] J. R. Millán, "Brain-computer interfaces," in Introduction to Neural Engineering for Motor Rehabilitation, D. Farina, W. Jensen, and M. Akay, Eds., pp. 237-251, John Wiley \& Sons, Hoboken, NJ, USA, 2013.

[2] N. Birbaumer and L. G. Cohen, "Brain-computer interfaces: communication and restoration of movement in paralysis," The Journal of Physiology, vol. 579, no. 3, pp. 621-636, 2007.

[3] D. J. McFarland and J. R. Wolpaw, "Brain-computer interfaces for communication and control," Communications of the ACM, vol. 54, no. 5, pp. 60-66, 2011.

[4] C. M. C. Allen, "Conscious but paralysed: releasing the lockedin," The Lancet, vol. 342, no. 8864, pp. 130-131, 1993.

[5] N. Birbaumer, F. Piccione, S. Silvoni, and M. Wildgruber, "Ideomotor silence: the case of complete paralysis and braincomputer interfaces (BCI)," Psychological Research, vol. 76, no. 2, pp. 183-191, 2012.

[6] T. M. Vaughan, D. J. McFarland, G. Schalk et al., "The wadsworth BCI research and development program: At home with BCI," IEEE Transactions on Neural Systems and Rehabilitation Engineering, vol. 14, no. 2, pp. 229-233, 2006.

[7] N. Birbaumer, N. Ghanayim, T. Hinterberger et al., "A spelling device for the paralysed," Nature, vol. 398, no. 6725, pp. 297-298, 1999.

[8] N. Neumann, T. Hinterberger, J. Kaiser, U. Leins, N. Birbaumer, and A. Kübler, "Automatic processing of self-regulation of slow cortical potentials: evidence from brain-computer communication in paralysed patients," Clinical Neurophysiology, vol.115, no. 3, pp. 628-635, 2004.

[9] A. Kübler, F. Nijboer, J. Mellinger et al., "Patients with ALS can use sensorimotor rhythms to operate a brain-computer interface," Neurology, vol. 64, no. 10, pp. 1775-1777, 2005.

[10] C. Neuper, G. R. Müller, A. Kübler, N. Birbaumer, and G. Pfurtscheller, "Clinical application of an EEG-based braincomputer interface: a case study in a patient with severe motor impairment," Clinical Neurophysiology, vol. 114, no. 3, pp. 399409, 2003.

[11] O. Bai, P. Lin, D. Huang, D. Fei, and M. K. Floeter, "Towards a user-friendly brain-computer interface: initial tests in ALS and PLS patients," Clinical Neurophysiology, vol. 121, no. 8, pp. 12931303, 2010.

[12] J. R. Wolpaw and D. J. McFarland, "Control of a twodimensional movement signal by a noninvasive brain-computer interface in humans," Proceedings of the National Academy of Sciences of the United States of America, vol. 101, no. 51, pp. 17849-17854, 2004. 
[13] S. Silvoni, C. Volpato, M. Cavinato et al., "P300-based braincomputer interface communication: evaluation and follow-up in amyotrophic lateral sclerosis," Frontiers in Neuroscience, vol. 3, no. 60, 2009.

[14] U. Hoffmann, J.-M. Vesin, T. Ebrahimi, and K. Diserens, "An efficient P300-based brain-computer interface for disabled subjects," Journal of Neuroscience Methods, vol. 167, no. 1, pp. 115-125, 2008.

[15] J. N. Mak, D. J. McFarland, T. M. Vaughan et al., "EEG correlates of P300-based brain-computer interface (BCI) performance in people with amyotrophic lateral sclerosis," Journal of Neural Engineering, vol. 9, no. 2, Article ID 026014, 2012.

[16] F. Nijboer, E. W. Sellers, J. Mellinger et al., "A P300-based brain-computer interface for people with amyotrophic lateral sclerosis," Clinical Neurophysiology, vol. 119, no. 8, pp. 1909-1916, 2008.

[17] F. Piccione, F. Giorgi, P. Tonin et al., "P300-based brain computer interface: reliability and performance in healthy and paralysed participants," Clinical Neurophysiology, vol. 117, no. 3, pp. 531-537, 2006.

[18] G. Pires, U. Nunes, and M. Castelo-Branco, "Statistical spatial filtering for a P300-based BCI: tests in able-bodied, and patients with cerebral palsy and amyotrophic lateral sclerosis," Journal of Neuroscience Methods, vol. 195, no. 2, pp. 270-281, 2011.

[19] E. W. Sellers and E. Donchin, "A P300-based brain-computer interface: initial tests by ALS patients," Clinical Neurophysiology, vol. 117, no. 3, pp. 538-548, 2006.

[20] G. Townsend, B. K. LaPallo, C. B. Boulay et al., "A novel P300-based brain-computer interface stimulus presentation paradigm: moving beyond rows and columns," Clinical Neurophysiology, vol. 121, no. 7, pp. 1109-1120, 2010.

[21] D. de Massari, C. A. Ruf, A. Furdea et al., "Brain communication in the locked-in state," Brain, vol. 136, no. 6, pp. 1989-2000, 2013.

[22] J. Polich, "Updating P300: an integrative theory of P3a and P3b," Clinical Neurophysiology, vol. 118, no. 10, pp. 2128-2148, 2007.

[23] J. Jin, B. Z. Allison, E. W. Sellers et al., "Optimized stimulus presentation patterns for an event-related potential EEG-based brain-computer interface," Medical and Biological Engineering and Computing, vol. 49, no. 2, pp. 181-191, 2011.

[24] E. W. Sellers, D. J. Krusienski, D. J. McFarland, T. M. Vaughan, and J. R. Wolpaw, "A P300 event-related potential braincomputer interface (BCI): the effects of matrix size and inter stimulus interval on performance," Biological Psychology, vol. 73, no. 3, pp. 242-252, 2006.

[25] M. S. Treder and B. Blankertz, "overt attention and visual speller design in an ERP-based brain-computer interface," Behavioral and Brain Functions, vol. 6, article 28, 2010.

[26] D. J. Krusienski, E. W. Sellers, D. J. McFarland, T. M. Vaughan, and J. R. Wolpaw, "Toward enhanced P300 speller performance," Journal of Neuroscience Methods, vol. 167, no. 1, pp. 1521, 2008.

[27] H. Cecotti, B. Rivet, M. Congedo et al., "A robust sensorselection method for P300 brain-computer interfaces," Journal of Neural Engineering, vol. 8, no. 1, Article ID 016001, 2011.

[28] M. Xu, H. Qi, L. Ma et al., "Channel selection based on phase measurement in P300-based brain-computer interface," PLoS ONE, vol. 8, no. 4, Article ID e60608, 2013.

[29] H. Cecotti and A. Gräser, "Convolutional neural networks for P300 detection with application to brain-computer interfaces," IEEE Transactions on Pattern Analysis and Machine Intelligence, vol. 33, no. 3, pp. 433-445, 2011.
[30] D. J. Krusienski, E. W. Sellers, F. Cabestaing et al., "A comparison of classification techniques for the P300 Speller," Journal of Neural Engineering, vol. 3, no. 4, article 007, pp. 299-305, 2006.

[31] N. V. Manyakov, N. Chumerin, A. Combaz, and M. M. Van Hulle, "Comparison of classification methods for P300 braincomputer interface on disabled subjects," Computational Intelligence and Neuroscience, vol. 2011, Article ID 519868, 12 pages, 2011.

[32] M. Kaper, P. Meinicke, U. Grossekathoefer, T. Lingner, and H. Ritter, "BCI competition 2003-data set IIb: support vector machines for the P300 speller paradigm," IEEE Transactions on Biomedical Engineering, vol. 51, no. 6, pp. 1073-1076, 2004.

[33] O. I. Khan, F. Farooq, F. Akram, M. T. Choi, S. M. Han, and T. Kim, "Robust extraction of P300 using constrained ICA for BCI applications," Medical and Biological Engineering and Computing, vol. 50, no. 3, pp. 231-241, 2012.

[34] H. Serby, E. Yom-Tov, and G. F. Inbar, "An improved P300-based brain-computer interface," IEEE Transactions on Neural Systems and Rehabilitation Engineering, vol. 13, no. 1, pp. 89-98, 2005.

[35] B. Rivet, A. Souloumiac, V. Attina, and G. Gibert, "xDAWN algorithm to enhance evoked potentials: application to braincomputer interface," IEEE Transactions on Biomedical Engineering, vol. 56, no. 8, pp. 2035-2043, 2009.

[36] A. Kübler, V. K. Mushahwar, L. R. Hochberg, and J. P. Donoghue, "BCI Meeting 2005-workshop on clinical issues and applications," IEEE Transactions on Neural Systems and Rehabilitation Engineering, vol. 14, no. 2, pp. 131-134, 2006.

[37] C. Zickler, A. Riccio, F. Leotta et al., "A brain-computer interface as input channel for a standard assistive technology software," Clinical EEG and Neuroscience, vol. 42, no. 4, pp. 236-244, 2011.

[38] G. Sparacino, S. Milani, E. Arslan, and C. Cobelli, "A Bayesian approach to estimate evoked potentials," Computer Methods and Programs in Biomedicine, vol. 68, no. 3, pp. 233-248, 2002.

[39] C. D’Avanzo, S. Schiff, P. Amodio, and G. Sparacino, "A Bayesian method to estimate single-trial event-related potentials with application to the study of the P300 variability," Journal of Neuroscience Methods, vol. 198, no. 1, pp. 114-124, 2011.

[40] J. M. Cedarbaum, N. Stambler, E. Malta et al., “The ALSFRS$\mathrm{R}$ : a revised ALS functional rating scale that incorporates assessments of respiratory function," Journal of the Neurological Sciences, vol. 169, no. 1-2, pp. 13-21, 1999.

[41] T. Joachims, "Making large-scale support vector machine learning practical," in Advances in Kernel Methods: Support Vector Learning, B. Schölkopf, C. J. Burges, and A. J. Smola, Eds., pp. 169-184, MIT Press, Cambridge, Mass, USA, 1999.

[42] A. Hyvärinen, "Fast and robust fixed-point algorithms for independent component analysis," IEEE Transactions on Neural Networks, vol. 10, no. 3, pp. 626-634, 1999.

[43] F. Beverina, F. Giorgi, S. Giove, F. Piccione, and S. Silvoni, "P300 off-line detection: a fuzzy-based support system," in Soft Computing Applications, A. Bonarini, F. Masulli, and G. Pasi, Eds., pp. 155-164, Springer, Berlin, Germany, 2003.

[44] S. Schiff, C. D’Avanzo, G. Cona et al., "Insight into the relationship between brain/behavioral speed and variability in patients with minimal hepatic encephalopathy," Clinical Neurophysiology, vol. 125, no. 2, pp. 287-297, 2014.

[45] S. Twomey, "The application of numerical filtering to the solution of integral equations encountered in indirect sensing measurements," Journal of the Franklin Institute, vol. 279, pp. 95-109, 1965. 
[46] G. de Nicolao, G. Sparacino, and C. Cobelli, "Nonparametric input estimation in physiological systems: problems, methods, and case studies," Automatica, vol. 33, no. 5, pp. 851-870, 1997.

[47] G. de Nicolao, G. Ferrari-Trecate, and G. Sparacino, "Fast spline smoothing via spectral factorization concepts," Automatica, vol. 36, no. 11, pp. 1733-1739, 2000.

[48] N. J. Hill, T. N. Lal, M. Schröder et al., "Classifying EEG and ECoG signals without subject training for fast BCI implementation: comparison of nonparalyzed and completely paralyzed subjects," IEEE Transactions on Neural Systems and Rehabilitation Engineering, vol. 14, no. 2, pp. 183-186, 2006.

[49] L. S. Snogdal, L. Folkestad, R. Elsborg et al., "Detection of hypoglycemia associated EEG changes during sleep in type 1 diabetes mellitus," Diabetes Research and Clinical Practice, vol. 98, no. 1, pp. 91-97, 2012. 


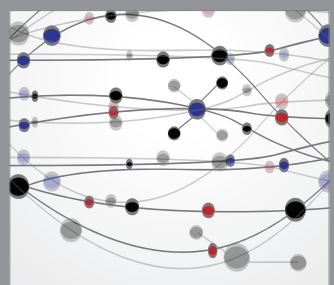

The Scientific World Journal
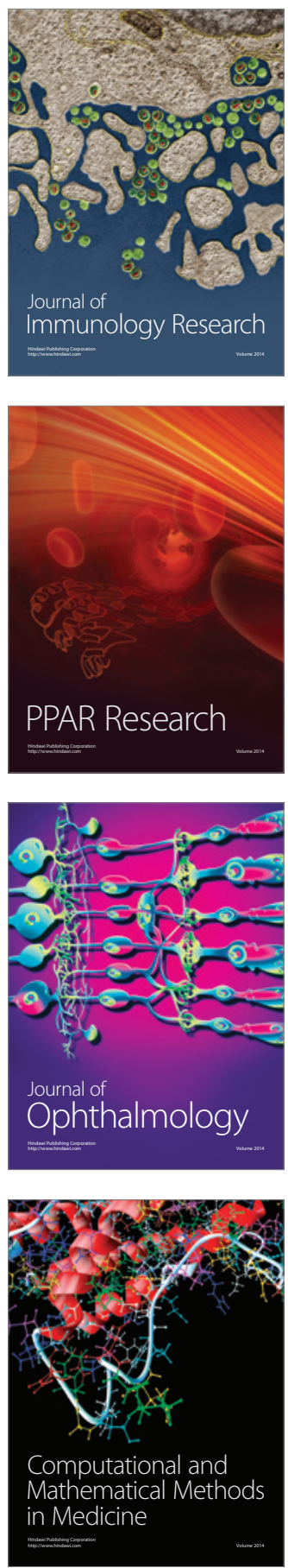

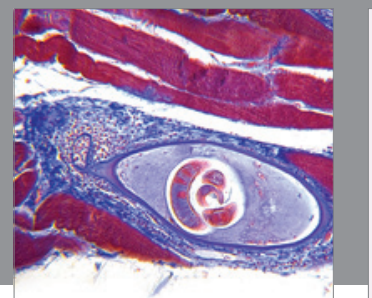

Gastroenterology

Research and Practice
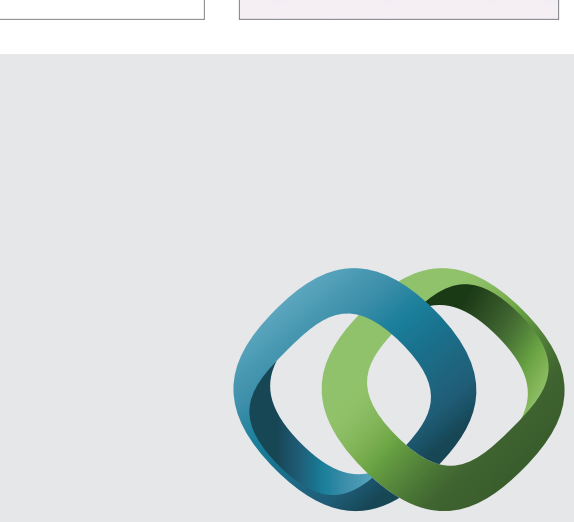

\section{Hindawi}

Submit your manuscripts at

http://www.hindawi.com
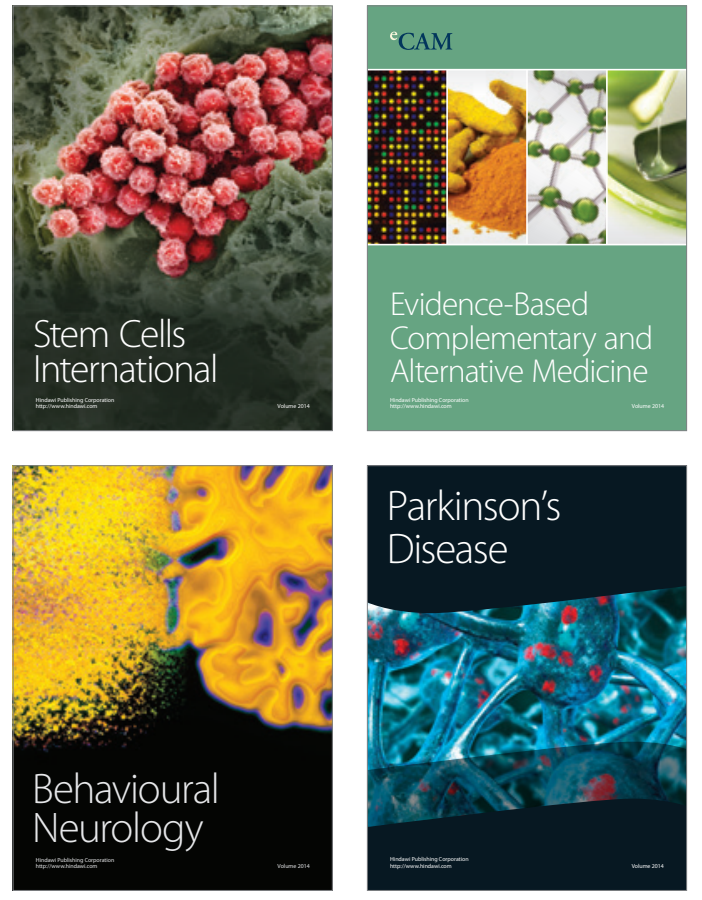
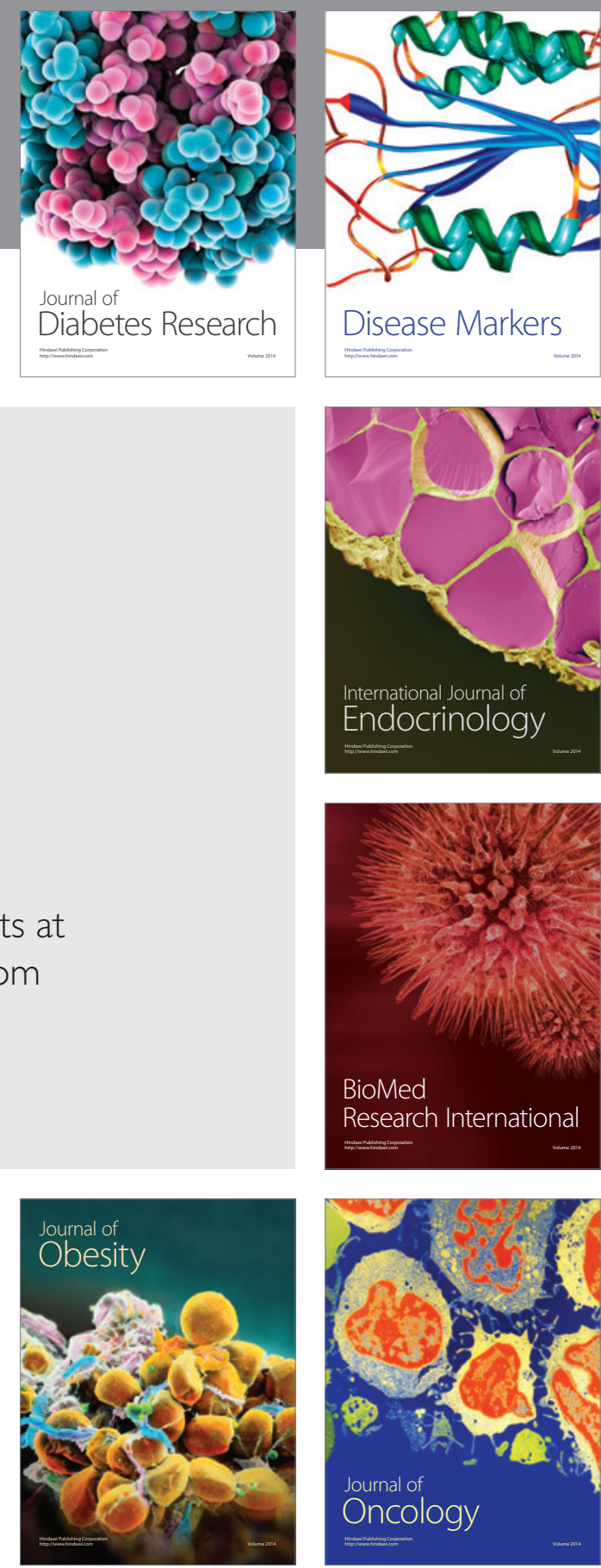

Disease Markers
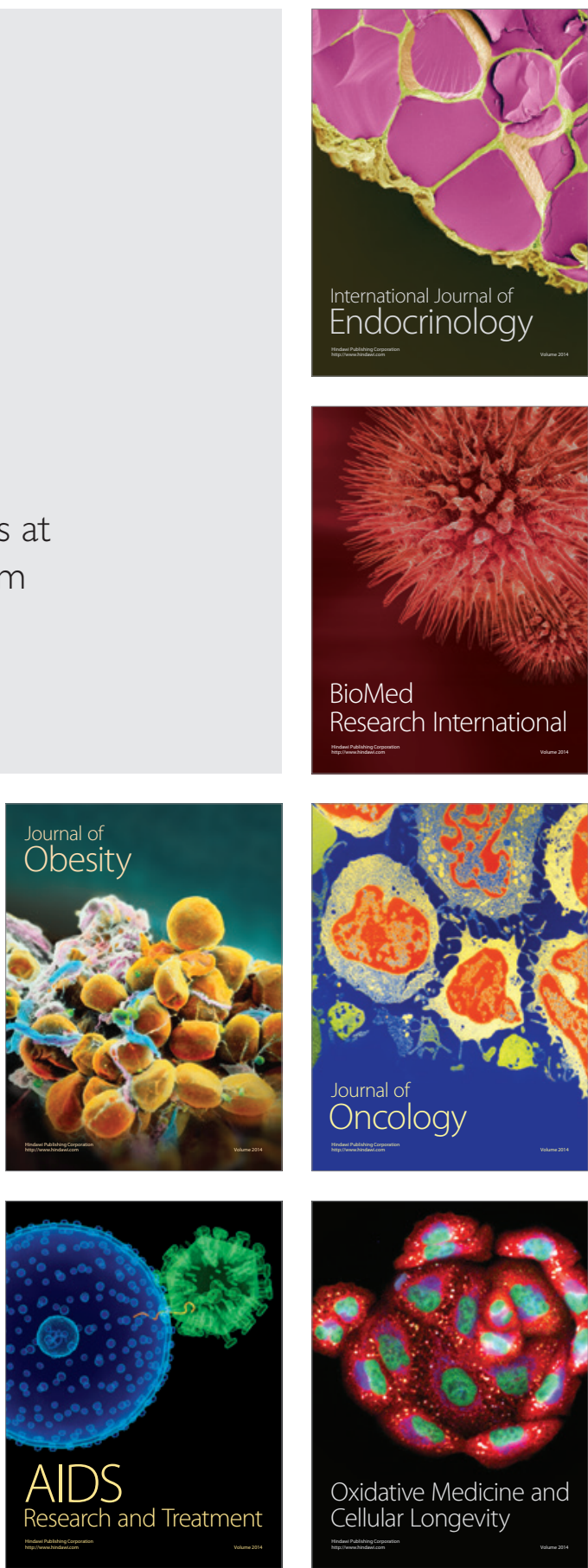